\title{
A Study based on Various Face Recognition Algorithms
}

\author{
Yukti Bakhshi \\ CTIEMT \\ Shahpur \\ Jalandhar, Punjab
}

\author{
Sukhvir Kaur \\ CTIEMT \\ Shahpur \\ Jalandhar, Punjab
}

\author{
Prince Verma \\ CTIEMT \\ Shahpur \\ Jalandhar, Punjab
}

\begin{abstract}
In recent years, the biometrics has achieved a great attention on a world level. A Biometric System operates by getting biometric information from a personal that extracts a feature set from the data which is acquired, and helps in comparing this feature set against the template stored in the database. There are biometric technologies which could either be physiological or behavioral. Face Recognition is having the importance to provide biometric authentication with easy image acquisition that can be used for online and offline applications. There are number of existing approaches for biometric facial recognition and classification. This paper gives a review on some of the common and reliable approaches which include PCA, LDA, SVM, SIFT, SURF, etc.
\end{abstract}

\section{General Terms}

Your general terms must be any term which can be used for general classification of the submitted material such as Pattern Recognition, Security, Algorithms et. al.

\section{Keywords}

Keywords are your own designated keywords which can be used for easy location of the manuscript using any search engines.

\section{INTRODUCTION}

Biometrics refers to the automatic identification of a person based on his or her physiological or behavioral characteristics. This identification method is preferred over traditional methods involving passwords and PINs for several reasons including the person to be identified is required to be physically present at the point of identification and/or identification based on biometric techniques obviates the need to remember a password and carry a token. [13] A Biometric System is a pattern recognition system which operates by acquiring the data from an individual and compares this feature set against the template set stored in the database. The human physical characteristics like face, hand geometry, fingerprints, voice, palm, signature and iris are known as "BIOMETRICS". One of the most important techniques is the face recognition.

\subsection{Physical Biometrics}

Physiological characteristics refers to a relatively stable human physical characteristics. Some of the examples include, but are not limited to fingerprint, face recognition, DNA, palm print, hand geometry, iris recognition, retina. The physiological characteristics are:

- Fingerprint: It analyzes the fingertip patterns.

- Facial recognition/face location: It helps in measuring facial characteristics.

- Hand geometry: It measures the shape of the hand.
- Iris scan: It analyzes the features of colored part of the eye.

- Retina scan: It analyzes the blood vessels in the eye.

- DNA: Universal human trait.

\subsection{Behavioral Biometrics}

Behavioral characteristics are related to the physical traits of a person. Some of the behavioral characteristics are as follows:

- Speaker recognition helps in analyzing the vocal behaviour.

- Signature recognition analyzes the signature dynamics.

- Keystroke recognition measures the time spacing between the typed words.

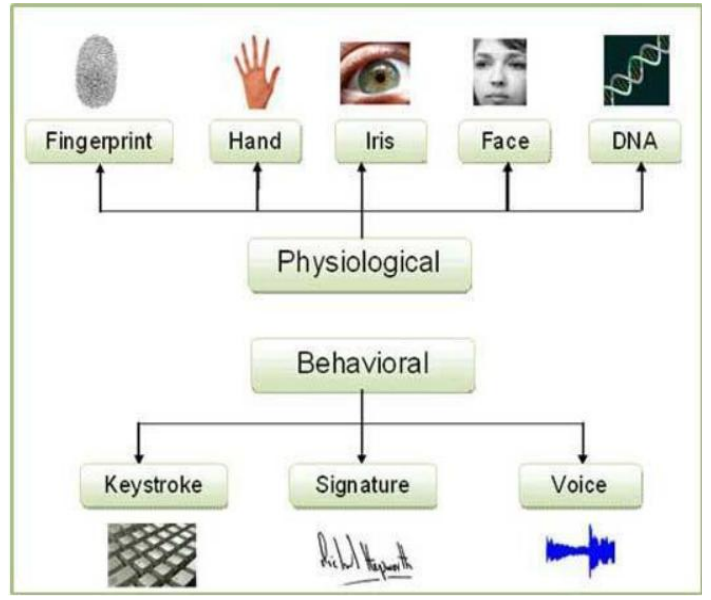

Fig 1: Types of Biometrics [5]

\section{FACE RECOGNITION}

Face recognition is one of most relevant applications of image analysis. Face Recognition is a task of identifying an already detected face and telling exactly who's it is and also deals with unique facial characteristics of human beings It can be applied in various challenging fields like video retrieval, security systems and identity authentication. It involves the pattern recognition and image processing. There are mainly two types of comparisons which are described as follows:

- Verification - When the system compares the given individual with the individual whom he says he is and gives a yes or no decision.

- Identification - When the system compares the given individual to all the other individuals stored in the database and gives a ranked list of matches.

Facial recognition methods involve a series of steps that are capturing, analyzing and comparing your face to a database of 
stored images. Below is the basic process that is used by the face recognition system to capture and compare images:

\subsection{Detection}

The recognition software searches the faces using the video camera when the system is attached to a video surveillance system. And if there is a face in the view, it is detected within a fraction of a second.

\subsection{Alignment}

Once the system has detected the face, then it determines the head's position, size and pose. For the system to register the face it needs to be turned at least 35 degrees towards the camera.

\subsection{Normalization}

For the image of the head to be registered and mapped into an appropriate size and pose, it is scaled and rotated. Normalization is performed despite of the head's location and distance from the camera. Light is not an issue in the normalization process.

\subsection{Representation}

After the normalization has been done, the system converts the facial data into a unique code. This coding process allows for easier representation and comparison of the newly acquired facial data to facial data which is already stored.

\subsection{Matching}

The newly acquired facial data is compared to the stored data and linked to at least one stored facial representation. The system decides if the features extracted from the newly acquired facial data are a match or not. If a score is above a predetermined threshold, a match is declared.

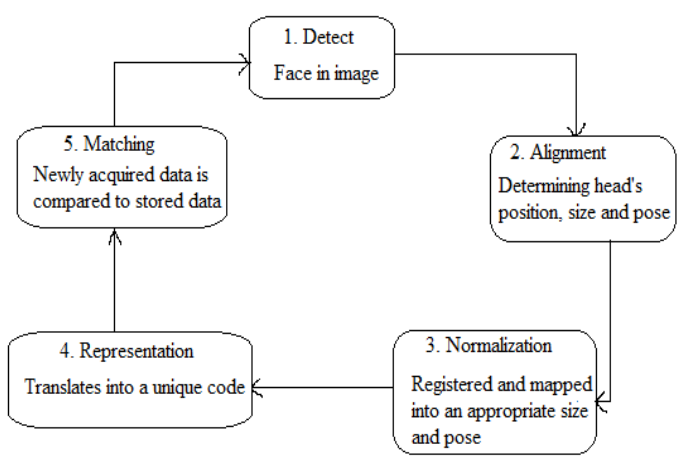

Fig 2: Basic process of Face Recognition system

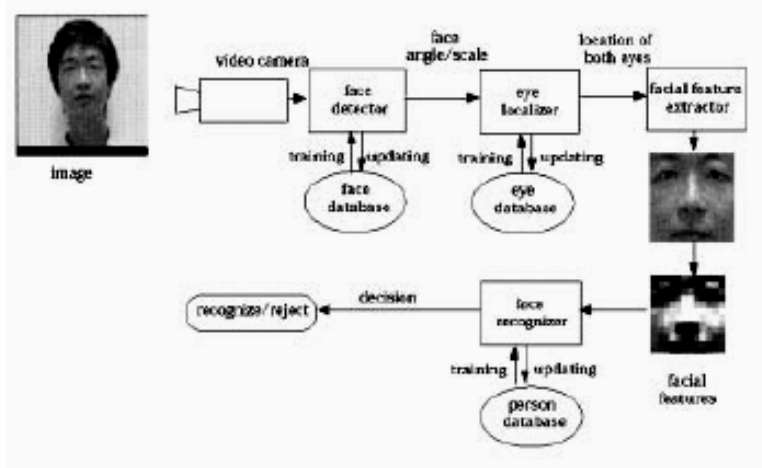

Fig 3: Face Recognition System Phases [8]

\section{FACE RECOGNITION ALGORITHMS \\ 3.1 PCA}

Principal Component Analysis commonly uses the eigenfaces in which the probe and gallery images must be the same size as well as normalized to line up the eyes and mouth of the subjects whining the images. Approach is then used to reduce the dimension of data by the means of image compression basics and provides most effective low dimensional structure of facial pattern. This reduction drops the unuseful information and decomposes the face structure into orthogonal (uncorrelated) components known as eigenfaces. Each face image is represented as weighted sum feature vector of eigenfaces which are stored in 1-D array. A probe image is compared against the gallery image by measuring the distance between their respective feature vectors then matching result has been disclosed. The main advantage of this technique is that it can reduce the data needed to identify the individual to $1 / 1000^{\text {th }}$ of the data presented. [12]

PCA solves the recognition problem within a representation space of lower dimension than image space. PCA is an eigenface method which helps in the reduction of the dimensionality of the original data space. But there is a disadvantage of PCA which says that recognition rate decreases under varying pose and illumination.

A face recognition system can be considered as a good system if we extract with the help of Principal Component Analysis and for recognition back propagation Neural Network are used. [12]

\subsection{LDA}

Linear Discriminant Analysis is an appearance based technique used for dimensionality reduction and recorded a great performance in face recognition. It provides us with a small set of features that carry the most relevant information for classification purposes.

LDA is a statistical approach for classifying samples of unknown classes based on training samples with known classes. This technique aims to maximum between-class (across users) variance and minimum within class (within user) variance. In these techniques a block represents a class, and there are a large variations between blocks but little variations within classes. It searches for those vectors in underlying space that best discriminate among classes (rather than those that best describe the data). More formally given a number of independent features relative to which the data is described. LDA creates a linear combination of these which yields the largest mean difference between desire classes.

Mathematically two measures are defined:

- One is called within-class scatter matrix which is given by-

$$
S_{w}=\sum_{j=1}^{c} \sum_{i=1}^{\bar{N}_{j}}\left(X_{i}^{j}-\mu_{j}\right)\left(X_{i}^{j}-\mu_{j}\right)^{T}
$$

- $\quad$ Other is called between class scatter matrix

$$
S_{b}=\sum_{j=1}^{c}\left(\mu_{j}-\mu\right)\left(\mu_{j}-\mu\right)^{T}
$$

LDA based algorithms performs better than PCA, but they suffer from the small-sample-size problem (SSS). 
Table 1. Comparison of PCA and LDA

\begin{tabular}{|c|c|}
\hline PCA & LDA \\
\hline $\begin{array}{c}\text { PCA is a dimensionality } \\
\text { reduction technique. }\end{array}$ & $\begin{array}{c}\text { LDA is a more particular } \\
\text { generative method. }\end{array}$ \\
\hline $\begin{array}{c}\text { PCA helps in feature } \\
\text { classification. }\end{array}$ & $\begin{array}{c}\text { LDA supports data } \\
\text { classification. }\end{array}$ \\
\hline $\begin{array}{c}\text { The shape and location of } \\
\text { original data sets changes } \\
\text { when transformed to a } \\
\text { different space. [6] }\end{array}$ & $\begin{array}{c}\text { LDA doesn't change the } \\
\text { location but only tries to } \\
\text { provide more class } \\
\text { separability and draw a } \\
\text { decision region between the } \\
\text { given classes. [6] }\end{array}$ \\
\hline $\begin{array}{c}\text { PCA calculates best } \\
\text { discriminating components } \\
\text { without knowledge about } \\
\text { groups. [6] }\end{array}$ & $\begin{array}{c}\text { LDA calculates best } \\
\text { discriminating components } \\
\text { about groups which are } \\
\text { defined by the client (user). } \\
\text { [6] }\end{array}$ \\
\hline \multicolumn{2}{|c|}{} \\
\hline
\end{tabular}

\subsection{SVM}

Support Vector Machine (SVM) is a binary classifier as a method for learning. SVM is a classification method that separate two data sets with maximum distance between them. The concept is to extend the spatial resolution around the margin by a conformal mapping, such that the divisibility between classes is increased. SVM cannot be applied directly when some of the features (face pixels) are occluded. In this case, values for those dimensions are unknown. SVM cannot be used when the feature vectors defining our samples have missing entries.

Support vector machines (SVMs) are formulated to solve a classical two class pattern recognition problem. [22]

\subsection{SIFT}

In 2004 Lowe, invents SIFT descriptor which is invariant to scale, rotation, affine transformation, noise, occlusions and is highly distinctive. SIFT features consist of four major stages in detection and representation; they are (1) finding scalespace extrema; (2) key point localization and filtering; (3) orientation assignment; (4) key point descriptor. The first stage is to construct the key points of images by using Difference-of-Gaussian (DoG) function. The second stage, candidate key points are restricted to sub-pixel accuracy and removed if found to be unreliable. The third stage represents the dominant orientations for each essential point of the images. The final stage constructs a descriptor for each key point location depends upon the image gradients in its local neighborhood. Then the SIFT descriptor is accepting the 128dimensional vector which used to identify the neighborhood around a pixel. The SIFT extracts the key points (locations and descriptors) for all the database images. Then given an altered image SIFT extracts the key point for that image and compares that point to the dataset. [2]
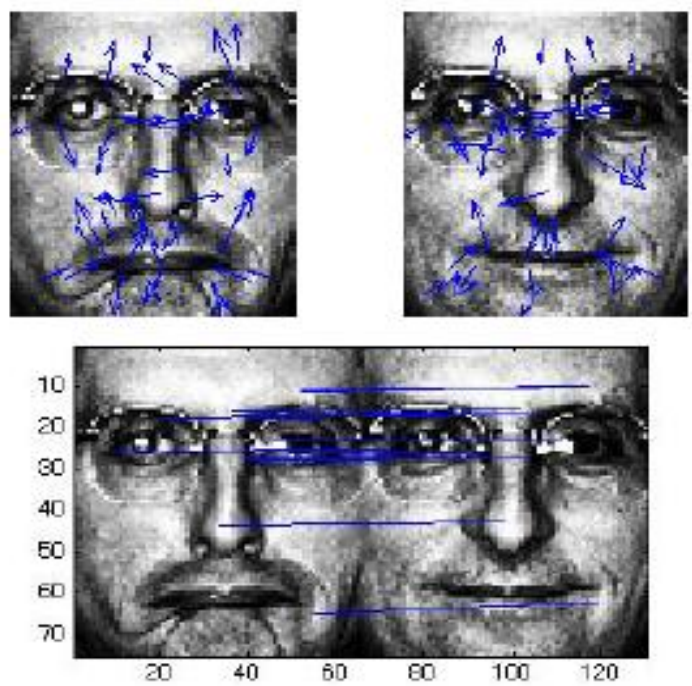

Fig 4: SIFT features on sample images and features matched in faces with expression variation. [20]

\subsection{SURF}

The SURF also extracts the key points from both the database images and the altered images. This method matches the key points between altered image and each database image. In 2008, H. Bay invents SURF descriptor which is invariant to a scale and in-plane rotation features. It consists of two stages such as interest point detector and interest point descriptor. In the first stage, locate the interest point in the image. Use the Hessian matrix to find the approximate detection. [2]

SURF is a scale and in-plane rotation invariant detector and descriptor. [16] SURF detectors are find the interest points in an image, and descriptors are used to extract the feature vectors at each interest point just as in SIFT. SURF uses Hessian-matrix approximation to locate the interest points instead of difference of Gaussians (DoG) filter used in SIFT. SURF as a descriptor uses the first-order Haar wavelet responses in $\mathrm{x}$ and $\mathrm{y}$, whereas the gradient is used by SIFT. SURF usually uses 64 dimensions in SURF to reduce the time cost for both feature matching and computation. SURF has three times better performance as compared to SIFT.
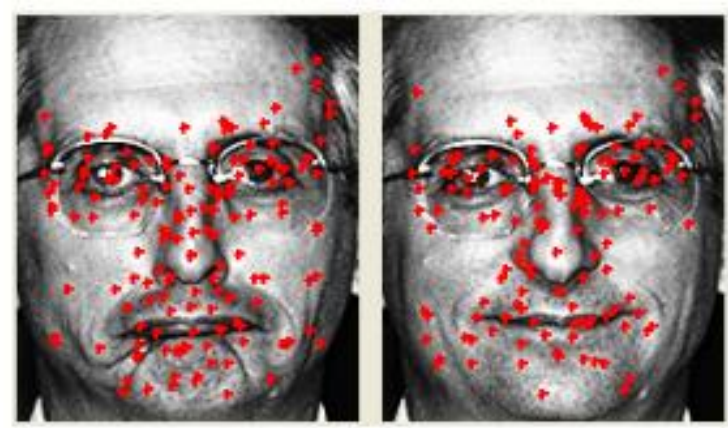

Fig 5: Interest points in face image [18] 


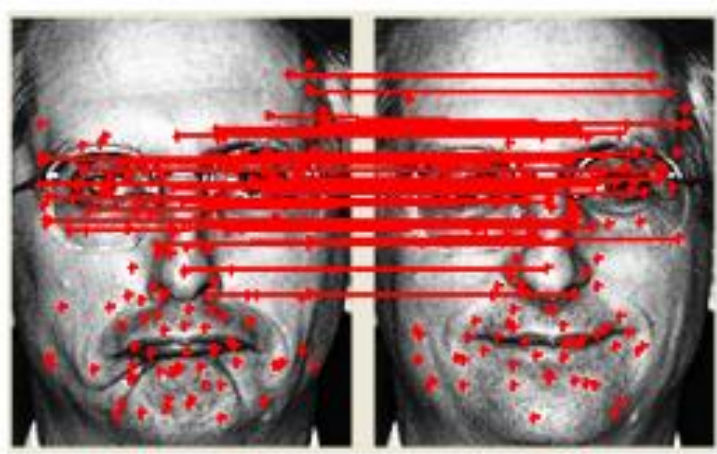

Fig 6: Example of point matching result [18]

Table 2. Comparison of SIFT and SURF

\begin{tabular}{|c|c|c|}
\hline & SIFT & SURF \\
\hline Scale Space & $\begin{array}{l}\text { DOG convolved } \\
\text { with difference } \\
\text { size images }\end{array}$ & $\begin{array}{l}\text { Difference size } \\
\text { box filter } \\
\text { convolved with } \\
\text { integral image }\end{array}$ \\
\hline $\begin{array}{c}\text { Key points } \\
\text { detection }\end{array}$ & $\begin{array}{l}\text { Local extrema } \\
\text { detection- Non- } \\
\text { maxima } \\
\text { suppression - } \\
\text { Eliminate edge } \\
\text { responses with } \\
\text { Hessian matrix }\end{array}$ & $\begin{array}{l}\text { Determine the } \\
\text { potential key } \\
\text { points with } \\
\text { Hessian matrix } \\
\text { and Non- } \\
\text { maximum } \\
\text { suppression } \\
\end{array}$ \\
\hline Orientation & $\begin{array}{l}\text { Image gradient } \\
\text { magnitudes and } \\
\text { orientations are } \\
\text { sampled around } \\
\text { the key point } \\
\text { location, using the } \\
\text { scale of the key } \\
\text { point to select the } \\
\text { level of Gaussian } \\
\text { blur of the image }\end{array}$ & $\begin{array}{c}\text { A sliding } \\
\text { orientation } \\
\text { window of size } \\
\text { pi/3 detects the } \\
\text { dominant } \\
\text { orientation of the } \\
\text { Gaussian } \\
\text { weighted Haar } \\
\text { wavelet responses } \\
\text { at every sample } \\
\text { point within a } \\
\text { circular } \\
\text { neighborhood } \\
\text { around the interest } \\
\text { point. [23] }\end{array}$ \\
\hline
\end{tabular}

\section{ACKNOWLEDGMENTS}

Our sincere thanks to the faculty of Computer Science Department at CTIEMT, Jalandhar who have contributed to the development of this paper and for their continued support and feedback.

\section{REFERENCES}

[1] Dong Li, Huiling Zhou and Kin-Man Lam "High Resolution Face Verification Using Pore-Scale Facial Features" IEEE transactions on image processing, Vol. 24, No. 8, pp 2317-2327, 2015.

[2] Priyanka, Dr. Yashpal Singh "A Study on Facial Feature Extraction and Facial Recognition Approaches" International Journal of computer Science and Mobile Computing, Vol. 4, pp 166-174, 2015.

[3] Vrushali Purandare and KT Talele "Efficient Heterogeneous Face Recognition using Scale Invariant Feature Transform" IEEE International Conference on Circuits, Systems, Communication and Information Technology Applications (CSCITA), Vol. 1, pp 305-310, 2014.
[4] Sriram Emarose and Pinnamaneni Bhanu Prasad "Face Recognition and Person Localization using SURF for Automated Attendance System" International Journal of Computer Applications, No. 8, pp 0975 - 8887, 2014

[5] Aditi Verma and Meha Khera "Comparative Study on Biometrics: a Review" International Journal of Advanced Research in Computer Science and Software Engineering , Vol. 4, Issue 5, pp 233-236, 2014.

[6] Vikram Solunke, Pratik Kudle, Abhijit Bhise, Adil Naik, Prof. J.R. Prasad "A Comparison between Feature Extraction Techniques for Face Recognition" International Journal of Emerging Research in Management \&Technology, Volume 3, pp 38-41, 2014.

[7] Ambika Ramchandra, Ravindra Kumar "Overview of Face Recognition System Challenges" International Journal of Scientific and Technology Research, Vol. 2, pp 234-236, 2013.

[8] R. Raghavendra, Bian Yang, K.B. Raja, C. Busch "A new perspective- Face Recognition with light-field camera" IEEE International Conference on Biometrics, pp 1-8, 2013.

[9] B.K. Bairagi, S.C. Das, A. Chatterjee, B. Tudu "Expressions invariant face recognition using SURF and Gabor features" IEEE Third International Conference on Emerging Applications of Information Technology, pp 170-173, 2012.

[10] Shungang Hua, Guopeng Chen, Honglei Wei and Qiuxin Jiang "Similarity measure for image resizing using SIFT feature" EURASIP Journal on Image and Video Processing, SPRINGER, No. 1, 2012.

[11] A. Bansal, K.Mehta, S. Arora "Face Recognition using PCA Algorithm and LDA" IEEE Second International Conference on Advanced Computing and Communication Technologies, pp 251-254, 2012.

[12] Aruni Singh, Sanjay Kumar Singh, Shrikant Tiwar "Comparison of face Recognition Algorithms on Dummy Faces" The International Journal of Multimedia \& Its Applications (IJMA) Vol.4, pp 121-135, 2012

[13] K.P. Tripathi "A Comparative Study of Biometric Technologies with Reference to Human Interface" International Journal of Computer Applications, Vol. 14, No.5, pp $0975-8887,2011$.

[14] Ergun Gumus, Niyazi Kilic, Ahmet Sertbas, Osman N. Ucan "Evaluation of face recognition techniques using PCA, wavelets and SVM" Elsevier Expert Systems with Applications, Vol. 37, pp 6404-6408, 2010.

[15] T.F. Karim, M.S.H. Lipu, M.L. Rahman, F. Sultana "Face Recognition using PCA based method" IEEE International Conference on Advanced Management Science, pp 158-162, 2010.

[16] Jia Hongjun, A.M. Martinez "Support Vector Machines in face recognition with occlusions" IEEE Conference on Computer Vision and Pattern Recognition, pp 136-141, 2009.

[17] M.O. Faruqe, M. Al Mehedi Hasan "Face Recognition using PCA and SVM" $3^{\text {rd }}$ International Conference on Anti-counterfeiting, Security and Identification in Communication, pp 97-101, 2009. 
[18] Geng Du, Fei Su, Anni Cai "Face Recognition using SURF features" Pattern Recognition and Computer Vision, Vol. 7496, 2009.

[19] Herbert Bay , Andreas Ess, Tinne Tuytelaars and Luc Van Gool "Speeded-Up Robust Features (SURF)" Elsevier Computer Vision and Image Understanding, Vol 110, pp 346-359, 2008.

[20] Jun Luo, Yong Ma, Erina Takikawa, Shihong Lao, Masato Kawade, Bao-Liang Lu "Person specific SIFT features for face recognition" IEEE International Conference on Acoustics, Speech and Signal Processing, pp 593-596, 2007.
[21] David G. Lowe "Distinctive Image Features from ScaleInvariant Keypoints" International Journal of Computer Vision, 2004

[22] P. Jonathon Phillips "Support Vector Machines Applied to Face Recognition" Advances in Neural Information Processing Systems, pp 803-809, 1999.

[23] https://www.quora.com/Image-Processing/Differencebetween-SURF-and-SIFT-where-and-when-to-use-thisalgo 